\title{
CpG oligodeoxynucleotide induces bone marrow precursor cells into myeloid-derived suppressor cells
}

\author{
JIE CHEN ${ }^{1,2}$, CHENGYU DENG $^{3}$, QINGMIN SHI $^{3}$, JUN JIANG $^{4}$, \\ YONGBO ZHANG ${ }^{4}$, WEI SHAN ${ }^{4}$ and WEIMIN SUN ${ }^{1}$
}

\begin{abstract}
${ }^{1}$ Department of Immunology, National Key Laboratory of Medical Immunology and Institute of Immunology, Second Military Medical University, Shanghai 200433; ${ }^{2}$ Department of Ophthalmology, 117th Hospital of PLA, Hangzhou, Zhejiang 310013; ${ }^{3}$ Department of Centre for Disease Prevention and Control, Chengdu Military Region, Chengdu, Sichuan 610021; ${ }^{4}$ Department of Pediatric Surgery, West China Hospital of Sichuan University, Chengdu, Sichuan 610041, P.R. China
\end{abstract}

Received December 29, 2012; Accepted July 17, 2013

DOI: $10.3892 / \mathrm{mmr} .2013 .1655$

\begin{abstract}
Dendritic cells (DCs) and myeloid-derived suppressor cells (MDSCs) perform a number of functions in different immunological settings. In standard in vitro experiments, DCs are produced from mouse bone marrow (BM) cells in the presence of granulocyte-macrophage colonystimulating factor (GM-CSF) and IL-4. Our previous study demonstrated that $\mathrm{BM}$ precursor cells could differentiate into MDSCs when co-cultured with poly (I:C). In the present study, BM precursor cells cultured in GM-CSF and IL-4 were treated with $\mathrm{CpG}$ oligodeoxynucleotide ( $\mathrm{CpG}$ ODN). We observed that $\mathrm{Gr}^{+} \mathrm{CD} 1 \mathrm{~b}^{+}$cells exhibiting MDSC functions accumulated in the co-culture system. A similar phenomenon was also observed in Listeria monocytogenes-infected mice. In conclusion, we demonstrated that prolonged CpG ODN stimulation could inhibit the development of DCs and induce the differentiation of BM precursor cells into MDSCs.
\end{abstract}

\section{Introduction}

Common myeloid progenitors (CMPs), which derive from hematopoietic stem cells, are capable of differentiating into immature myeloid cells (IMCs). In pathological conditions, including cancer, various infectious diseases, sepsis, trauma, bone marrow (BM) transplantation and some autoimmune disorders, a partial inhibition of the differentiation of IMCs into mature myeloid cells results in the expansion of this population. Importantly, the activation of these cells in a

Correspondence to: Professor Weimin Sun, Department of Immunology, National Key Laboratory of Medical Immunology and Institute of Immunology, Second Military Medical University, 800 Xiangyin Road, Shanghai 200433, P.R. China

E-mail: weiminsunchina@163.com

Key words: dendritic cells, myeloid-derived suppressor cells, $\mathrm{CpG}$ oligodeoxynucleotide, Listeria monocytogenes pathological context results in the upregulated expression of several immune suppressive factors, including arginase, inducible nitric oxide synthase (iNOS), nitric oxide (NO) and reactive oxygen species (ROS). Together, these factors lead to the expansion of the IMC population which possesses immunosuppressive activity. These cells are collectively known as myeloid-derived suppressor cells (MDSCs) (1).

Dendritic cells (DCs) and MDSCs perform different functions in the immune response. DCs are important in initiating the innate and adaptive immune responses $(2,3)$. DCs are also capable of inducing a T-cell immune response by presenting the foreign antigen, upregulating costimulatory molecules and releasing proinflammatory cytokines following microbial or inflammatory activation $(4,5)$. The immune response is regulated by various cell types, including Foxp $3^{+}$and IL-10producing regulatory $\mathrm{T}$ cells $(6)$, regulatory $\mathrm{DCs}(7,8)$ and MDSCs. MDSCs have been demonstrated to regulate the immune response through arginase, iNOS $(9,10)$, ROS (11) and the induction of Foxp $3^{+}$regulatory cells $(12,13)$. The development and expansion of MDSCs is associated with cancer, infection and autoimmunity (1).

Lipopolysaccharide (LPS), the ligand of Toll-like receptor 4, is able to either induce the maturation of DCs (4) or expand the $\mathrm{Grl}^{+} \mathrm{CD} 11 \mathrm{~b}^{+}$cell population in the spleen during polymicrobial sepsis (14). Also, our previous study demonstrated that BM precursor cells are capable of differentiating into MDSCs when co-cultured with poly (I:C) (15).

However, whether other conserved structural patterns of microbial components, including $\mathrm{CpG}$ oligodeoxynucleotide (CpG ODN) possess a similar ability to induce MDSCs has yet to be reported. In the present study, we used CpG ODN to investigate the balance between the development of MDSCs and DCs from the same BM precursor cells. We observed that sustained stimulation with CpG ODN leads to the development and expansion of MDSCs and inhibition of DC development.

\section{Materials and methods}

Mice. Male and female wild-type C57BL/6 mice, 5-6 weeks of age, were purchased from the Chinese Academy of 
Sciences (Shanghai, China). DO11.10 OVA $323-339^{-s p e c i f i c ~}$ TCR-transgenic mice with a C57BL/6 background were obtained from The Jackson Laboratory (Bar Harbor, ME, USA). Mice were housed in a specific pathogen-free facility for all experiments. All animal experiments were undertaken in accordance with the National Institutes of Health 'Guide for the Care and Use of Laboratory Animals' (NIH Publication no. 85-23, National Academy Press, Washington, DC, revised 1996), with approval by the Laboratory Animal Center and Ethics Committee of the Second Military Medical University (Shanghai, China).

Reagents. Recombinant mouse granulocyte-monocyte colonystimulating factor (GM-CSF), interleukin 4 (IL-4) and ELISA kits for murine interleukin 1 (IL-1), interleukin 6 (IL-6), interleukin 12p40 (IL-12p40), matrix metalloproteinase 9 (MMP-9), tumor necrosis factor $\alpha$ (TNF- $\alpha$ ), IFN- $\gamma$ and transforming growth factor $\beta$ (TGF- $\beta$ ) were purchased from R\&D Systems (Minneapolis, MN, USA). Fluorescein-conjugated monoclonal antibodies (mAbs) to CD4, CD11b, Iab, CD40, CD80, CD86 and isotype control were purchased from Santa Cruz Biotechnology, Inc. (Santa Cruz, CA, USA). Fluoresceinconjugated $\mathrm{mAbs}$ to $\mathrm{Grl}$ were obtained from eBioscience (San Diego, CA, USA). 7-Aminoactinomycin D (7-AAD), LPS and BSA were purchased from Sigma-Aldrich (St. Louis, MO, USA). CpG-A DNA (ODN 2216) was purchased from HyCult Biotech (Uden, The Netherlands).

Preparation of DCs from mouse BM precursor cells and pre-treated DCs. BM mononuclear cells were prepared from mouse (5-6 weeks old) femur BM suspensions by depletion of red blood cells. The cells were cultured at a density of $2 \times 10^{6}$ cells $/ \mathrm{ml}$ in 6 -well plates in RPMI-1640 medium supplemented with $10 \%$ fetal calf serum (FCS), $10 \mathrm{ng} / \mathrm{ml}$ of recombinant mouse GM-CSF and $1 \mathrm{ng} / \mathrm{ml}$ of recombinant mouse IL-4. Nonadherent cells were gently washed out on day 4 of culture. On day 5 , the dendritic-proliferating clusters were collected and purified by anti-CD11c microbeads as immature DCs (imDCs). ImDCs were stimulated with LPS $(100 \mathrm{ng} / \mathrm{ml})$ for a further 2 days and then collected as mature DCs (mDCs). CpG ODN (6 $\mu \mathrm{g} / \mathrm{ml})$ was added to the BM-DC culture system $\left(1 \times 10^{6}\right)$ on day 0 (long stimulation) or on day 5 (short stimulation) (16).

Analysis of phagocytic ability. Cells from different groups were incubated at $37^{\circ} \mathrm{C}$ for $4 \mathrm{~h}$ with FITC-conjugated OVA to a final concentration of $100 \mu \mathrm{g} / \mathrm{ml}$ in RMPI-1640 medium containing $10 \%$ FCS, and were washed twice with ice-cold PBS (pH 7.2) containing $0.1 \% \mathrm{NaN}_{3}$ and $0.5 \%$ BSA. The cells were resuspended in chilled PBS for immediate analysis by flow cytometry.

Assay for cytokines and NO. The aforementioned cytokines in the supernatant of the DC system were assayed with the corresponding ELISA kits. NO production was assayed by measurement of the nitrite concentration using the Griess assay.

Assay for antigen (Ag)-specific $C D 4^{+} T$-cell response. In order to assay the $\mathrm{Ag}$-specific $\mathrm{CD} 4^{+} \mathrm{T}$-cell response, splenic $\mathrm{CD}^{+}$
T cells from DO11.10 OVA $_{323-339}$-specific TCR-transgenic mice were obtained. Cells were positively selected with antiCD4-coated microbeads (Miltenyi Biotech, Minneapolis, MN, USA) by magnetic-activated cell sorting (MACS) and then co-cultured with treated DCs as indicated in the presence of $\mathrm{OVA}_{323-339}$ peptide at a ratio of 1:10 (DC:T) in round-bottom 96-well plates $\left(1 \times 10^{5} \mathrm{~T}\right.$ cells $/ 200 \mu \mathrm{l} /$ well) for 5 days. The proliferation of $\mathrm{T}$ cells was analyzed by double staining with anti-CD4 ${ }^{+}$and 7-AAD cells were counted by fluorescenceactivated cell sorting (FACS).

Listeria monocytogenes (L. monocytogenes) and experimental infection of mice. L. monocytogenes strain 10403s was purchased from American Type Culture Collection (Manassas, VA, USA) and the bacterial stocks were stored in aliquots that were maintained at $-70^{\circ} \mathrm{C}$. The titers were checked for each experiment to confirm the number of viable injected bacteria. The level of infection in the various experimental groups was determined by enumerating the L. monocytogenes $\mathrm{CFU}$ in the liver and spleen as described previously (17). In order to confirm whether sustained L. monocytogenes infection was capable of causing the accumulation of MDSCs, 10 wild-type C57BL/6 mice were challenged i.v. with a dose of $103 \mathrm{CFU}$ of L. monocytogenes strain 10403s. Mice were monitored daily and euthanized 1 or 5 days later. Following isolation of the spleen and depletion of red blood cells, cells were labeled with anti-CD11c, anti-CD11b and anti-Gr1 mAbs for flow cytometry analysis.

Statistical analysis. Comparisons between the experimental groups and relevant control were performed by ANOVA. $\mathrm{P}<0.05$ was considered to indicate a statistically significant difference.

\section{Results}

BM precursor cells demonstrate a distinct phenotype and cytokine profile following long stimulation with CpG ODN. Mouse BM-derived DCs were generated by culturing cells in GM-CSF and IL-4 (7,18-20). In the standard DC-induced protocol, on day $0, \mathrm{BM}$ precursor cells were washed from the femurs of mice and then were co-cultured with GM-CSF and IL-4 for 5 days. On day 5, the dendritic-proliferating clusters were collected and purified by anti-CD11c microbeads as imDCs. In this study, CpG ODN was added to the BM-DC culture system on day 0 (long stimulation) or on day 5 (short stimulation). On day 6 , the phenotype and function of cells were analyzed. We observed that cells in the long stimulation group (L-group) demonstrated a distinct morphology with fewer cell colonies (Fig. 1A). We additionally observed that cells in the L-group exhibited a distinct phenotype with lower expression levels of Iab, CD40, CD80 and CD86, and cells in the short stimulation group (S-group) exhibited higher expression levels of these costimulated molecules (Fig. 1B). We then harvested the day 6 cells from each group separately. Cells were washed in PBS 3 times and were then seeded in a 24-well plate. Supernatants from each well were collected for cytokine and NO analysis $24 \mathrm{~h}$ later (day 7). Compared with the cytokine profile of the S-group and control, cells in the L-group secreted lower levels of IL-1 $\beta$, IL-6, IL-12p40, 
A

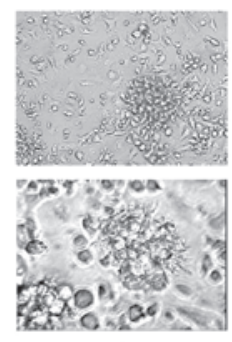

Control

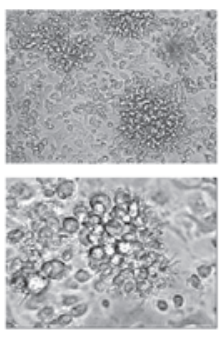

short stimulation

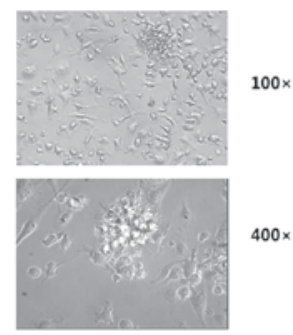

long stimulation
B
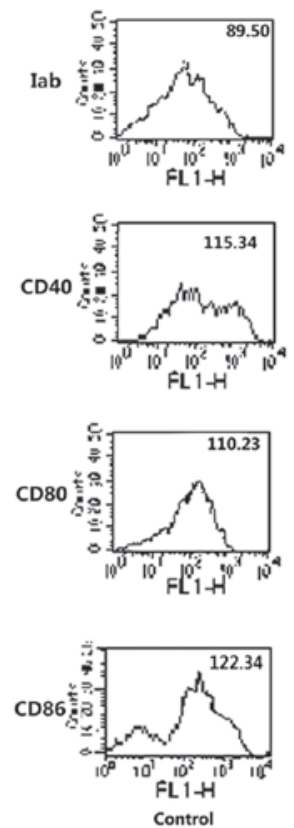
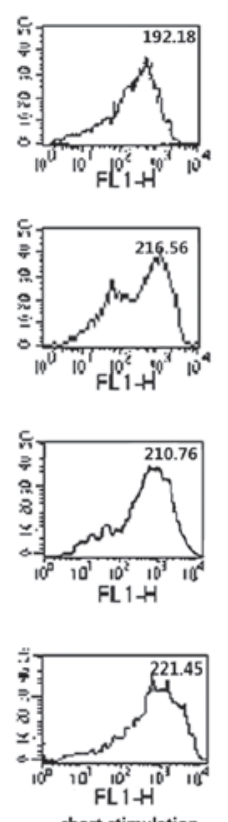

short stimulation
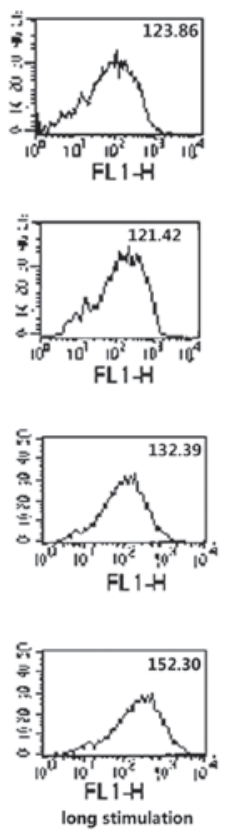

C
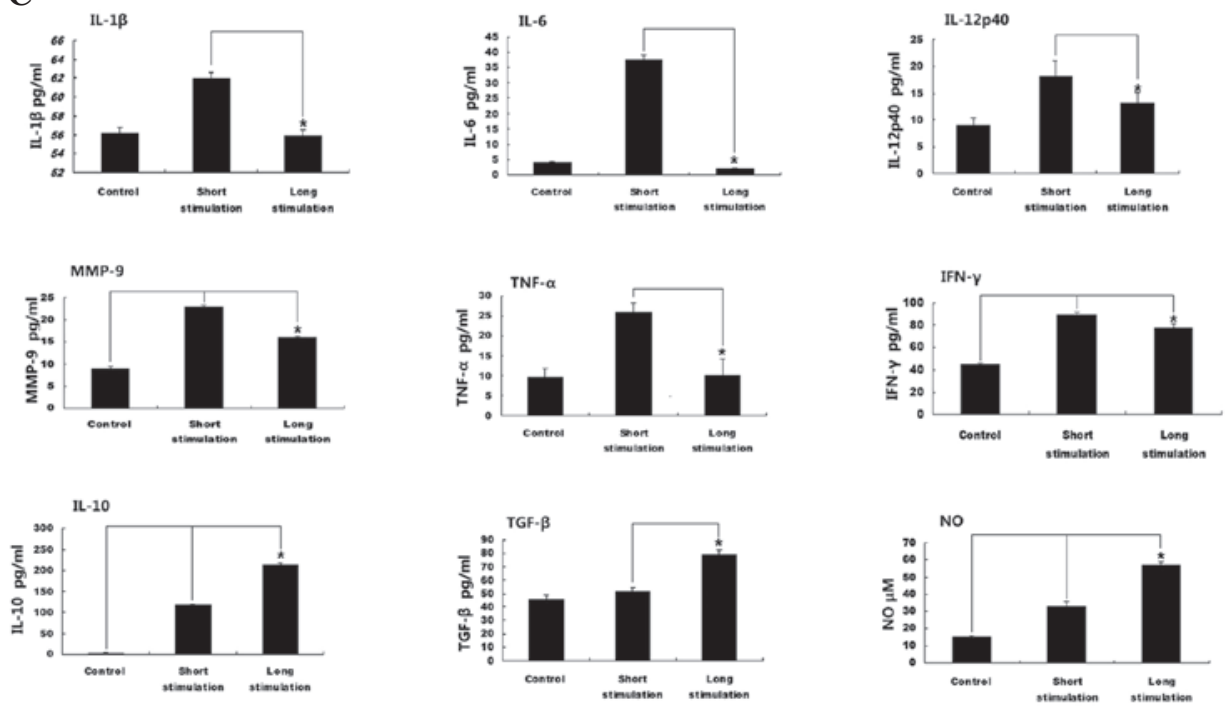

Figure 1. CpG ODN induced the differentiation of bone marrow precursor cells into a number of different cell types. (A) Distinct morphology of cells from each group. Bone marrow mononuclear cells were prepared from mouse (5-6 weeks old) femur bone marrow suspensions by depletion of red blood cells, and cells from the same mouse were then divided equally into 3 groups for further treatment. The long stimulation group was cultured with GM-CSF, IL-4 and CpG ODN $(6 \mu \mathrm{g} / \mathrm{ml})$ on day 0 ; the short stimulation group was cultured with GM-CSF and IL-4 on day 0 , and CpG ODN (6 $\mu \mathrm{g} / \mathrm{ml}) \mathrm{was}$ added on day 5 ; the control group was cultured with GM-CSF and IL-4 on day 0, without CpG ODN. The cells were then observed under a microscope (magnification x100 or x400) on day 6. Following long stimulation, the cells demonstrated a distinct morphology with fewer cell colonies. (B) The phenotype of cells from each group. Cells from the control, short stimulation and long stimulation groups were labeled with Ab to Iab, CD40, CD80 and CD86 for phenotypic analysis by flow cytometry. Numbers in histograms indicate the geometric mean fluorescence intensity. (C) Different cytokine profiles and NO expression levels of each group. On day 6, cells from each group were collected and washed in PBS 3 times, and then were seeded in a 24 -well plate at a density of $1.0 \times 10^{6} \mathrm{ml} / \mathrm{well}$. Supernatants from each well were collected $24 \mathrm{~h}$ later (day 7 ) for analysis of cytokine and NO production. IL-1 $\beta$, IL-6, IL-12p40, MMP-9, TNF- $\alpha$, INF- $\gamma$, IL-10, TGF- $\beta$ and NO produced by cells from each group were measured by ELISA or Griess assay for $24 \mathrm{~h}$. Results are presented as the mean \pm SD of triplicate wells. ${ }^{*} \mathrm{P}<0.05$. CpG ODN, CpG oligodeoxynucleotide; GM-CSF, granulocyte-macrophage colony-stimulating factor; NO, nitric oxide; MMP, matrix metalloproteinase. 
A

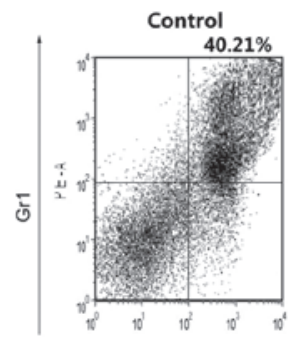

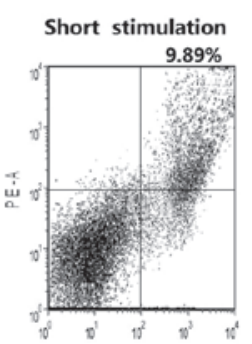

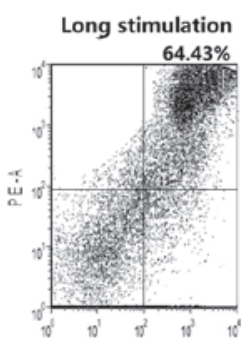

CD11b

\section{B}
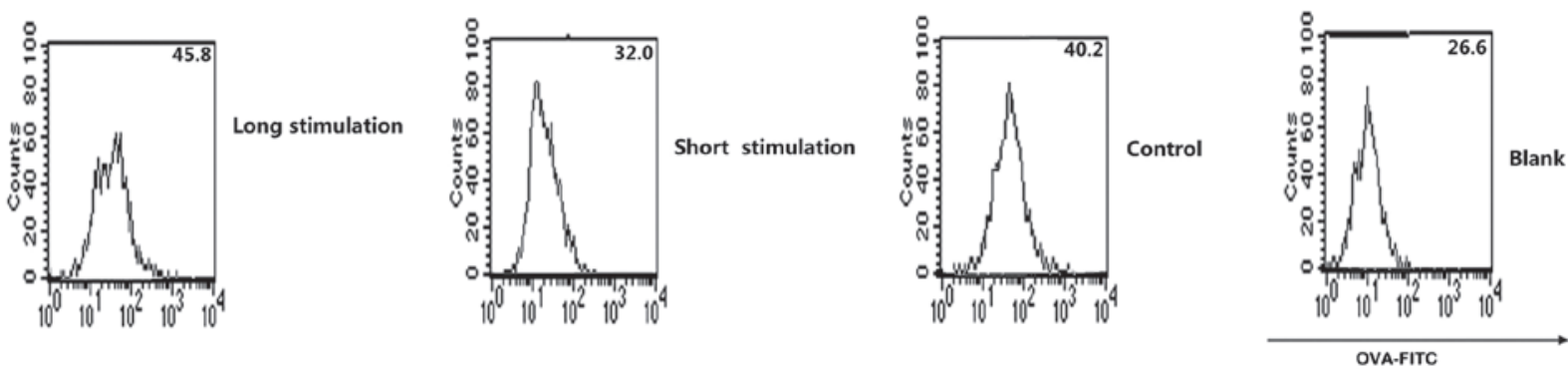

C

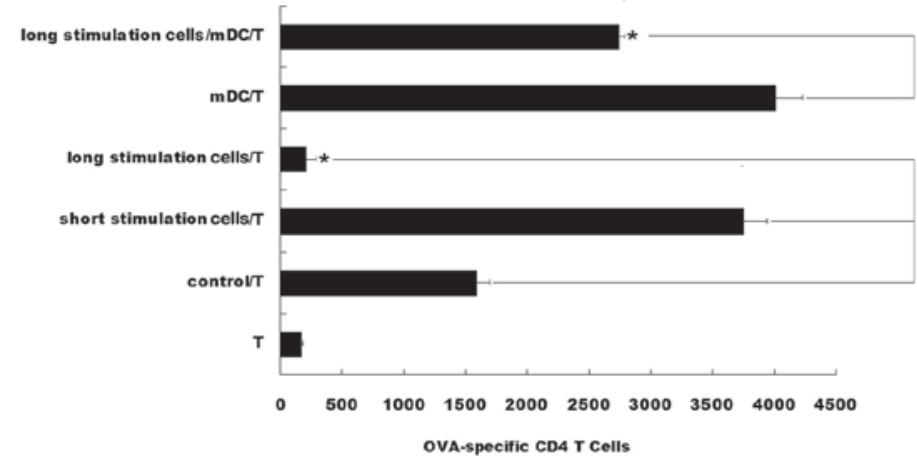

Figure 2. Cells in the long stimulation group demonstrated an enhanced phagocytic ability and inhibited CD4+ T-cell proliferation. (A) On day 6 , the percentage of $\mathrm{Grl}^{+} \mathrm{CD} 11 \mathrm{~b}^{+}$cells in different groups was analyzed by flow cytometry. The percentage of $\mathrm{Grl}^{+} \mathrm{CD} 11 \mathrm{~b}^{+}$double-positive cells in the long stimulation group was $64.43 \%$ compared with $40.21 \%$ (control) and $9.89 \%$ (short stimulation group). (B) Phagocytic ability was assessed for OVA-FITC phagocytosis by flow cytometry. Numbers in histograms indicate the geometric mean fluorescence of test samples. (C) $\mathrm{CD}^{+}{ }^{+} \mathrm{T}$ cells from DO11.10 OVA $\mathrm{O}_{323-339}$-specific $(\mathrm{TCR}-$ transgenic xC57BL/6) F1 hybrid mice were co-cultured with cells from each group. Five days later, the total number of viable $\mathrm{CD}^{+} \mathrm{T}$ cells $\left(\mathrm{CD} 4^{+}\right.$7AAD) in each well were measured by flow cytometry. Results are presented as the mean \pm SD of triplicate wells. " $\mathrm{P}<0.05$. 7-AAD, 7-aminoactinomycin D; mDCs, mature dendritic cells.

MMP-9 and TNF- $\alpha$, and higher levels of IFN- $\gamma$, IL-10, TGF- $\beta$ and NO (Fig. 1C). Altogether, these data demonstrate that long stimulation with $\mathrm{CpG}$ ODN may exert a strong effect on the development of DCs from BM cells.

Phagocytic ability and stimulation of T-cell proliferation. Since cells in the CpG ODN L-group demonstrated higher expression levels of CD11b, and secreted higher levels of IL-10, TGF- $\beta$ and NO (Fig. 1C), the phenotypes of cells from all groups were analyzed. An increase in the number of $\mathrm{Grl}^{+} \mathrm{CD} 11 \mathrm{~b}^{+}$cells in the $\mathrm{CpG}$ ODN L-group was observed (Fig. 2A). Therefore, we hypothesize that cells in the L-group may have transformed into MDSCs as a result of interaction with CpG ODN. The phagocytic ability of cells in each group was additionally analyzed and it was observed that cells in the L-group exhibited an enhanced phagocytic ability (Fig. 2B). The ability of DCs to stimulate an antigen-specific T-cell response was also examined. Cells in the L-group demonstrated a reduced ability to stimulate the proliferation of OVA-specific CD4 ${ }^{+} \mathrm{T}$ cells compared with the S-group and control. Significantly, when cells of the L-group were added to the $\mathrm{mDCs} / \mathrm{CD}^{+} \mathrm{T}$-cell co-culture system, in vitro $\mathrm{T}$-cell proliferation was partially suppressed (Fig. 2C). As the long stimulation cells exhibited a $\mathrm{Grl}^{+} \mathrm{CD} 11 \mathrm{~b}^{+}$phenotype and the ability to suppress the proliferation of $\mathrm{T}$ cells, we hypothesize that the $\mathrm{Grl}^{+} \mathrm{CD} 11 \mathrm{~b}^{+}$cells that accumulate in the L-group may be MDSCs.

Sustained L. monocytogenes infection causes the accumulation of MDSCs. CpG ODN is a short single-stranded synthetic DNA and CpG motif present in bacterial DNA and an example of a pathogen-associated molecular pattern (PAMP) (21-24). We examined whether sustained bacterial infection in vivo is capable of leading to the accumulation of MDSCs. Previous studies revealed that Listeria infection elicits a similar spectrum of cytokine production to CpG ODN $(25,26)$. Thus, we infected C57BL/6 mice with L. monocytogenes for 1 or 5 days. We observed that L. monocytogenes (Listeria) infection may 
$\mathbf{A}$

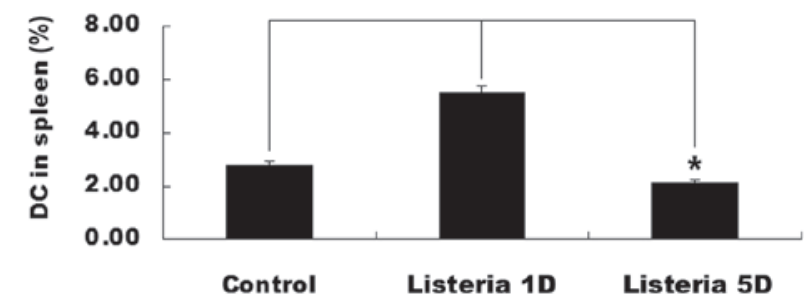

B

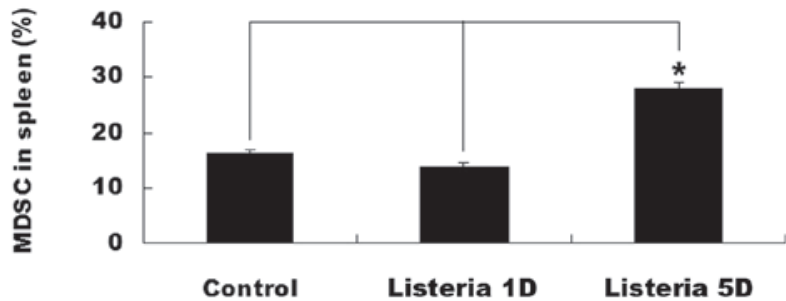

Figure 3. Sustained L. monocytogenes infection induced the proliferation of MDSCs and inhibited the expansion of DCs in vivo. Ten C57BL/6 mice, 8-10 weeks of age, were challenged i.v. with a dose of $103 \mathrm{CFU}$ of L. monocytogenes strain 10403s. Mice were monitored daily. After 1 or 5 days, the spleen was isolated for DC and MDSC phenotype analysis. Following isolation of the spleen and depletion of red blood cells, cells were labeled with anti-CD11c, anti-CD11b and anti-Gr1 mAbs for flow cytometry analysis. CD11c-positive cells were counted as DCs; Gr1 and CD11b double-positive cells were counted as MDSCs. The percentage of DCs and MDSCs were calculated by FACS software. (A) Percentage of DCs in the spleen on day 1 and 5. (B) Percentage of MDSCs in the spleen on day 1 and 5. Results were presented as the mean \pm SD of 10 mice. " $\mathrm{P}<0.05$. MDSCs, myeloid-derived suppressor cells; DCs, dendritic cells; L. monocytogenes, Listeria monocytogenes; mAbs, monoclonal antibodies; FACS, fluorescence-activated cell sorting; VSV, vesicular stomatitis virus.

upregulate the percentage of DCs and downregulate the percentage of MDSCs in the spleen due to a 1-day infection, and may downregulate the percentage of DCs and upregulate the percentage of MDSCs in the spleen due to a 5-day infection (Fig. 3). These results indicate that a prolonged infection may lead to an increase in the number of MDSCs and a decrease in the number of DCs, which is similar to the result induced by CpG ODN.

\section{Discussion}

The accumulation of MDSCs is observed in numerous pathological conditions, including cancer, bacterial infection, acute and chronic inflammation, traumatic stress, surgical sepsis and transplantation (1). In these diverse types of pathological conditions, various factors are involved, including cyclooxygenase-2 (COX2), prostaglandins (27-29), stem-cell factor (SCF) (27), macrophage colony-stimulating factor (M-CSF), IL-6 (30), GM-CSF (29) and vascular endothelial growth factor (VEGF) (31).

In our previous study, we added poly (I:C) into the standard DC-generated system whereby DCs were generated by co-culturing with GM-CSF and IL-4 (7,18-20). Following 5 days of stimulation, we observed the accumulation of MDSCs (15). In the present study, we observed similar phenomena following long stimulation with CpG ODN. Previous studies (14) and our data (not shown) also indicated that LPS is capable of expanding the $\mathrm{Grl}^{+} \mathrm{CD} 11 \mathrm{~b}^{+}$cell population and inhibiting DC development. It is useful to compare each of these three PAMPs, as these three TLR ligands are capable of expanding the $\mathrm{Gr}^{+} \mathrm{CD} 11 \mathrm{~b}^{+}$cell population and inhibiting DC development. Treatment of myeloid precursor cells with LPS led to a partial activation as indicated by NO release that allows a transient suppressive activity. It has been demonstrated that combined LPS/IFN- $\gamma$ signaling, as opposed to its components alone, is capable of activating myeloid precursor cells to differentiate into functionally suppressive MDSCs, which impairs their developmental potential into DCs (32). Our data suggests that poly (I:C) and CpG ODN induce myeloid precursor cells to differentiate into MDSCs, and the difference between poly (I:C) and CpG ODN is that a short and long stimulation of $\mathrm{CpG}$ ODN induces the production of high levels of IFN- $\gamma$. In the present study, CpG ODN class A was used, which exhibits potent immunostimulatory effects on plasmacytoid DCs (pDCs), and IFN- $\gamma$ is its key cytokine. In adult mice, pDCs are produced constantly in the $\mathrm{BM}$ and migrate from the BM to lymph nodes, mucosal-associated lymphoid tissues and spleen in steady-state conditions. Furthermore, CMPs are capable of differentiating into mDCs and $\mathrm{pDC}$ in culture and in vivo $(33,34)$. Based on the evidence above, we hypothesize that some pDCs may have mixed differentiation; however, further investigation is required.

There are at least three factors in our culture system: IL-4, GM-CSF and CpG ODN. GM-CSF is an essential growth factor in the maturation of DCs. GM-CSF is important in the negative regulation of the immune response through the induction of myeloid suppressor $\mathrm{Grl}^{+} \mathrm{CD} 11 \mathrm{~b}^{+}$cells $(29,35)$. Although the exact role of GM-CSF has yet to be identified, the system with or without $\mathrm{CpG}$ ODN may lead to different scenarios following long stimulation with $\mathrm{CpG}$ ODN, which stimulates the development and expansion of MDSCs.

A systemic expansion of MDSCs was observed in infection-like polymicrobial sepsis in mice (14), infection with helminths (36-38) and Candida albicans (39). We also observed the accumulation of MDSCs and decreased levels of DCs in the spleen following sustained infection with L. monocytogenes. It has been reported that $\mathrm{CpG}$ ODN may also induce resistance to L. monocytogenes infection following a 
single injection of CpG ODN (26), which is consistent with our data.

In conclusion, we demonstrated that BM precursor cells differentiate into MDSCs and not DCs following long stimulation with CpG ODN and sustained L. monocytogenes infection. Our data may provide novel insights into the mechanism of MDSC development during infection.

\section{Acknowledgements}

The authors appreciate the assistance of Dr Xuetao Cao from the National Key Laboratory of Medical Immunology and Dr Bing Yu from The Department of Cell Biology. The study was supported by grants from the National Natural Science Foundation of China (nos. 30872313 and 30271232)

\section{References}

1. Gabrilovich DI and Nagaraj S: Myeloid-derived suppressor cells as regulators of the immune system. Nat Rev Immunol 9: 162-174, 2009.

2. Steinman RM: The dendritic cell system and its role in immunogenicity. Annu Rev Immunol 9: 271-296, 1991.

3. Steinman RM and Banchereau J: Taking dendritic cells into medicine. Nature 449: 419-426, 2007.

4. Banchereau J and Steinman RM: Dendritic cells and the control of immunity. Nature 392: 245-252, 1998.

5. Steinman RM and Nussenzweig MC: Avoiding horror autotoxicus: the importance of dendritic cells in peripheral $\mathrm{T}$ cell tolerance. Proc Natl Acad Sci USA 99: 351-358, 2002.

6. Littman DR and Rudensky AY: Th17 and regulatory T cells in mediating and restraining inflammation. Cell 140: 845-858, 2010

7. Zhang M, Tang H, Guo Z, et al: Splenic stroma drives mature dendritic cells to differentiate into regulatory dendritic cells. Nat Immunol 5: 1124-1133, 2004.

8. Liu Q, Zhang C, Sun A, Zheng Y, Wang L and Cao X: Tumoreducated CD11bhighIalow regulatory dendritic cells suppress $\mathrm{T}$ cell response through arginase I. J Immunol 182: 6207-6216, 2009.

9. Bronte V and Zanovello P: Regulation of immune responses by L-arginine metabolism. Nat Rev Immunol 5: 641-654, 2005.

10. Rodríguez PC and Ochoa AC: Arginine regulation by myeloid derived suppressor cells and tolerance in cancer: mechanisms and therapeutic perspectives. Immunol Rev 222: 180-191, 2008.

11. Kusmartsev S, Nefedova Y, Yoder D and Gabrilovich DI: Antigen-specific inhibition of CD8+ T cell response by immature myeloid cells in cancer is mediated by reactive oxygen species. J Immunol 172: 989-999, 2004.

12. Yang R, Cai Z, Zhang Y, Yutzy WH 4th, Roby KF and Roden RB: CD80 in immune suppression by mouse ovarian carcinoma-associated Gr-1+CD11b+ myeloid cells. Cancer Res 66: 6807-6815, 2006.

13. Huang B, Pan PY, Li Q, et al: Gr-1+CD115+ immature myeloid suppressor cells mediate the development of tumor-induced $\mathrm{T}$ regulatory cells and T-cell anergy in tumor-bearing host. Cancer Res 66: 1123-1131, 2006.

14. Delano MJ, Scumpia PO, Weinstein JS, et al: MyD88-dependent expansion of an immature GR-1(+)CD11b(+) population induces $\mathrm{T}$ cell suppression and Th2 polarization in sepsis. J Exp Med 204: 1463-1474, 2007.

15. Liu C, Zhang C, Lu H, et al: Poly(I:C) induce bone marrow precursor cells into myeloid-derived suppressor cells. Mol Cell Biochem 358: 317-323, 2011.

16. Tsujimoto H, Efron PA, Matsumoto T, et al: Maturation of murine bone marrow-derived dendritic cells with poly(I:C) produces altered TLR-9 expression and response to CpG DNA. Immunol Lett 107: 155-162, 2006.

17. Harty JT and Bevan MJ: Specific immunity to Listeria monocytogenes in the absence of IFN gamma. Immunity 3: 109-117, 1995.

18. Tang H, Guo Z, Zhang M, Wang J, Chen G and Cao X: Endothelial stroma programs hematopoietic stem cells to differentiate into regulatory dendritic cells through IL-10. Blood 108: 1189-1197, 2006.
19. Xia S, Guo Z, Xu X, Yi H, Wang Q and Cao X: Hepatic microenvironment programs hematopoietic progenitor differentiation into regulatory dendritic cells, maintaining liver tolerance. Blood 112: 3175-3185, 2008.

20. Li Q, Guo Z, Xu X, Xia S and Cao X: Pulmonary stromal cells induce the generation of regulatory DC attenuating T-cellmediated lung inflammation. Eur J Immunol 38: 2751-2761, 2008.

21. Yamamoto S, Yamamoto T, Kataoka T, Kuramoto E, Yano O and Tokunaga T: Unique palindromic sequences in synthetic oligonucleotides are required to induce IFN [correction of INF] and augment IFN-mediated [correction of INF] natural killer activity. J Immunol 148: 4072-4076, 1992.

22. Krieg AM, Yi AK, Matson S, et al: CpG motifs in bacterial DNA trigger direct B-cell activation. Nature 374: 546-549, 1995.

23. Klinman DM, Yi AK, Beaucage SL, Conover J and Krieg AM: $\mathrm{CpG}$ motifs present in bacteria DNA rapidly induce lymphocytes to secrete interleukin 6, interleukin 12, and interferon gamma. Proc Natl Acad Sci USA 93: 2879-2883, 1996.

24. Hemmi H, Takeuchi O, Kawai T, et al: A Toll-like receptor recognizes bacterial DNA. Nature 408: 740-745, 2000.

25. Harty JT, Lenz LL and Bevan MJ: Primary and secondary immune responses to Listeria monocytogenes. Curr Opin Immunol 8: 526-530, 1996.

26. Krieg AM, Love-Homan L, Yi AK and Harty JT: CpG DNA induces sustained IL-12 expression in vivo and resistance to Listeria monocytogenes challenge. J Immunol 161: 2428-2434, 1998.

27. Pan PY, Wang GX, Yin B, et al: Reversion of immune tolerance in advanced malignancy: modulation of myeloid-derived suppressor cell development by blockade of stem-cell factor function. Blood 111: 219-228, 2008.

28. Sinha P, Clements VK, Fulton AM and Ostrand-Rosenberg S: Prostaglandin E2 promotes tumor progression by inducing myeloid-derived suppressor cells. Cancer Res 67: 4507-4513, 2007.

29. Serafini P, Carbley R, Noonan KA, Tan G, Bronte V and Borrello I: High-dose granulocyte-macrophage colony-stimulating factor-producing vaccines impair the immune response through the recruitment of myeloid suppressor cells. Cancer Res 64: 6337-6343, 2004.

30. Bunt SK, Yang L, Sinha P, Clements VK, Leips J and Ostrand-Rosenberg S: Reduced inflammation in the tumor microenvironment delays the accumulation of myeloid-derived suppressor cells and limits tumor progression. Cancer Res 67: 10019-10026, 2007.

31. Gabrilovich D, Ishida T, Oyama T, et al: Vascular endothelial growth factor inhibits the development of dendritic cells and dramatically affects the differentiation of multiple hematopoietic lineages in vivo. Blood 92: 4150-4166, 1998.

32. Greifenberg V, Ribechini E, Rössner S and Lutz MB: Myeloid-derived suppressor cell activation by combined LPS and IFN-gamma treatment impairs DC development. Eur J Immunol 39: 2865-2876, 2009.

33. D'Amico A and Wu L: The early progenitors of mouse dendritic cells and plasmacytoid predendritic cells are within the bone marrow hemopoietic precursors expressing Flt3. J Exp Med 198: 293-303, 2003.

34. Karsunky H, Merad M, Cozzio A, Weissman IL and Manz MG: Flt3 ligand regulates dendritic cell development from Flt3+ lymphoid and myeloid-committed progenitors to Flt3+ dendritic cells in vivo. J Exp Med 198: 305-313, 2003.

35. Bronte V, Apolloni E, Cabrelle A, et al: Identification of a $\mathrm{CD} 11 \mathrm{~b}(+) / \mathrm{Gr}-1(+) / \mathrm{CD} 31(+)$ myeloid progenitor capable of activating or suppressing CD8(+) T cells. Blood 96: 3838-3846, 2000.

36. Terrazas LI, Walsh KL, Piskorska D, McGuire E and Harn DA Jr: The schistosome oligosaccharide lacto-N-neotetraose expands Gr1(+) cells that secrete anti-inflammatory cytokines and inhibit proliferation of naive CD4(+) cells: a potential mechanism for immune polarization in helminth infections. J Immunol 167: 5294-5303, 2001

37. Gómez-García L, López-Marín LM, Saavedra R, Reyes JL, Rodríguez-Sosa M and Terrazas LI: Intact glycans from cestode antigens are involved in innate activation of myeloid suppressor cells. Parasite Immunol 27: 395-405, 2005.

38. Brys L, Beschin A, Raes G, et al: Reactive oxygen species and 12/15-lipoxygenase contribute to the antiproliferative capacity of alternatively activated myeloid cells elicited during helminth infection. J Immunol 174: 6095-6104, 2005.

39. Mencacci A, Montagnoli C, Bacci A, et al: CD80+Gr-1+ myeloid cells inhibit development of antifungal Th1 immunity in mice with candidiasis. J Immunol 169: 3180-3190, 2002. 